Gut, 1969, 10, 173-179

\title{
The act of vomiting in man
}

\author{
K. LUMSDEN AND W. S. HOLDEN ${ }^{1}$ \\ From the Radcliffe Infirmary, Oxford
}

The mechanism of vomiting in mammals is highly complex, and some aspects of it are not well understood despite the many experimental studies which have been made, particularly in cats and dogs. For example, there is still room for debate about the gastric and intestinal movements during nausea, about the role of the stomach in vomiting, and about the mechanism of opening of the cardia.

The reported studies of the act of vomiting in man are comparatively few. Most of these were fluoroscopic studies of persons vomiting after a bismuth or barium meal and were made many years ago with $x$-ray apparatus which would today be regarded as primitive. It was unsuitable for taking radiographs during the act because the long exposure times would have resulted in blurring from movement.

We ourselves, while performing routine barium meal examinations with modern $x$-ray apparatus, have seen patients become nauseated, start retching, and even sometimes actually vomit. We have observed the movements which take place in the duodenum, stomach, and lower oesophagus, and have taken radiographs of these organs before, during, and after vomiting. In the present paper we report three selected cases which illustrate the mechanism of vomiting when the stomach is normal.

\section{CASE REPORTS}

CASE 1 A married woman, aged 54 years, complained of nausea and vomiting of recent onset. Before examination she had taken Telepaque which outlined the biliary tree, and barium examination of the oesophagus and stomach with the patient standing showed normal appearances on fluoroscopy.

After the duodenal bulb had filled and a little barium had passed on into the second and third parts of the duodenum, a $12 \times 10$ in. radiograph was exposed (Fig. 1). The patient was already experiencing nausea at this stage of the examination and she soon began retching. Two further $12 \times 10$ in. radiographs were taken in quick succession (Figs. 2 and 3). At the time when the last of the series of three films was exposed, the patient had actually started to vomit; the cardia had dilated and barium was already passing up from the stomach into the oesophagus.

${ }^{1}$ Present address: Torbay Hospital, Torquay, Devon.
Interpretation of the radiographs The three radiographs constitute a record of the act of vomiting in this patient, and from a detailed analysis of them we have been able to form an opinion of the movements which occurred in the proximal duodenum, the stomach, and the lower oesophagus.

The first radiograph (Fig. 1), taken while the patient was nauseated and before retching started, is a true posteroanterior view; and the shadow of the gallbladder, which is unusually situated in that it is in the midline, is superimposed upon the fifth lumbar vertebra and the upper sacrum. The second part of the duodenum is contracted and the traces of barium which outline its mucosal folds are seen overlapping the gallbladder. The duodenal bulb is distended with barium while the pylorus is closed, and both lie just to the left of the midline with the pylorus at the level of the lumbo-sacral disc. The gastric antrum is moderately distended and its lowest part lies a little below the level of the fundus of the gallbladder. The middle part of the stomach is more contracted than either the upper or lower parts, and is represented by linear shadows of barium between longitudinal folds of mucosa. In the gastric fundus there is a small air bubble. The cardia is shut. The lower oesophagus is empty and appears in the original radiograph as fine linear shadows superimposed on the spine.

The second radiograph (Fig. 2) was taken while the patient was retching. As she was in a left anterior oblique position, the gallbladder has been projected to the right of the spine and is partly superimposed on the right ilium. The exposure seems to have been made at the precise moment when the cardia opened, as a blunt projection at the upper end of the lesser curvature marks the site of the cardia but there is no barium in the lower oesophagus. However, as the air bubble previously shown in the fundus has disappeared, the cardia may have opened at an earlier stage.

Comparison of the second radiograph with the first shows remarkable differences. The duodenal bulb, previously distended, has emptied, and the barium it contained appears to have passed back into the stomach, since the second part of the duodenum is still empty. The lower half of the stomach is now shown without much barium in it, whereas the upper half has become much more distended. Indeed, in the upper part of the stomach the diameter of the gastric lumen has nearly doubled, and it is evident that barium has been displaced from the lower part of the stomach into the upper part.

Further details seen in Fig. 2 are as follows. The pylorus is closed and the prepyloric region contains a 


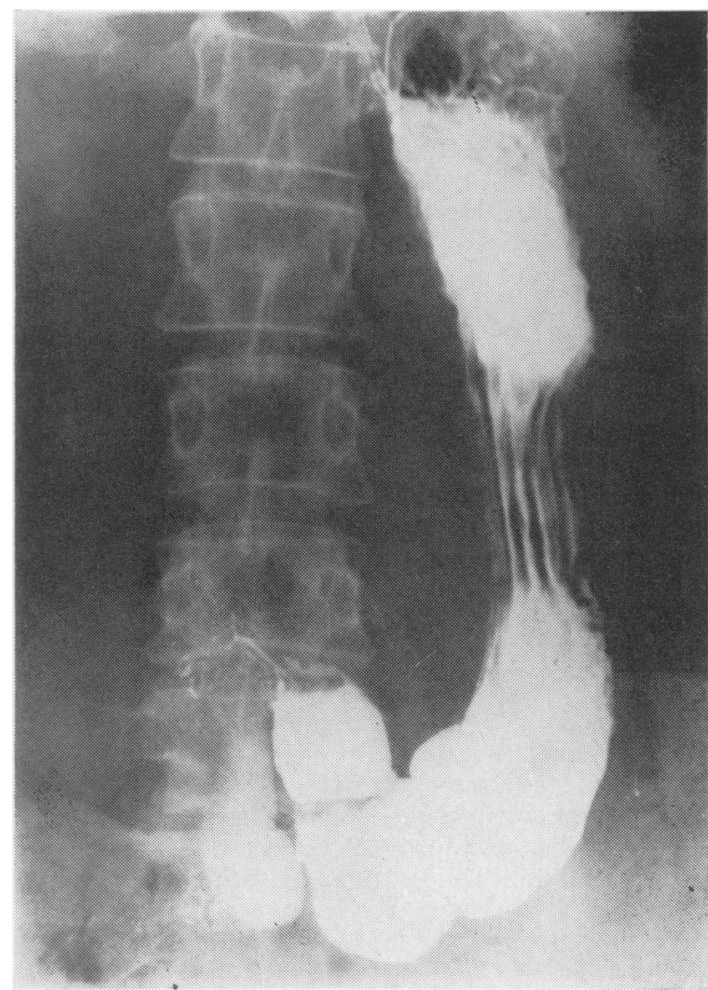

FIG. 1 .

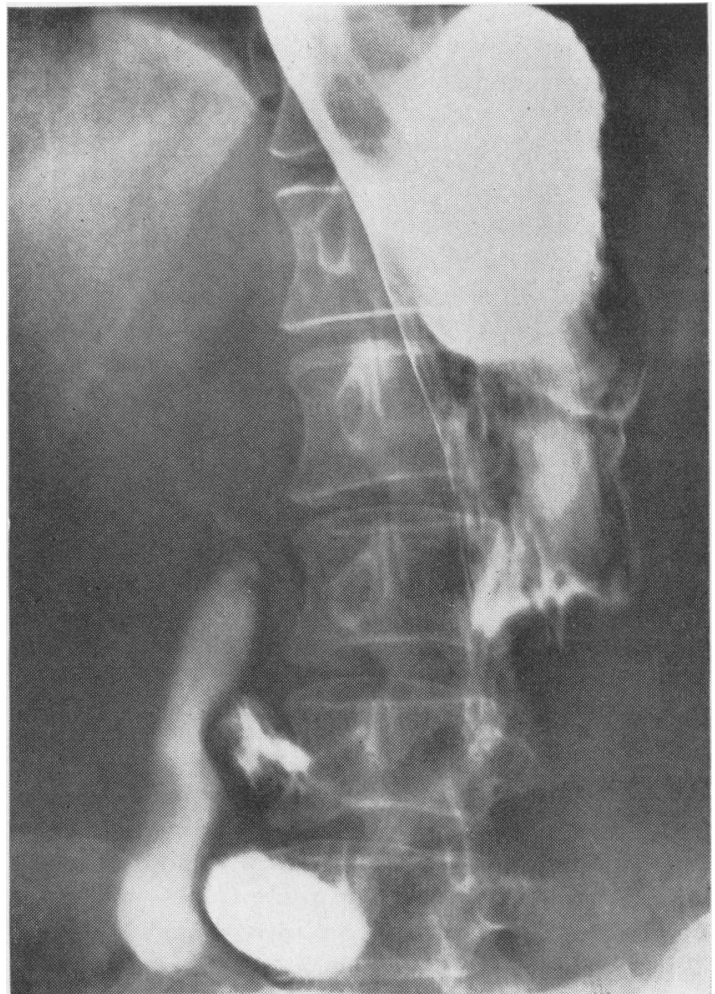

FIG. 3 .

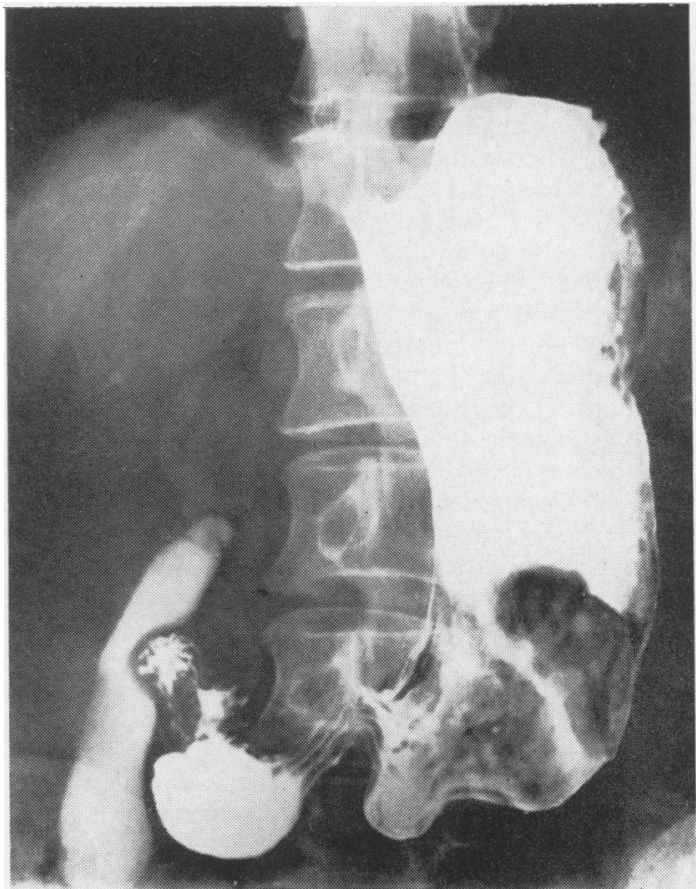

FIG. 1. Case 1. First radiograph, taken erect before retching began. The gallbladder shadow is superimposed on the spine, the duodenal bulb is distended with barium, and the stomach has not yet begun to contract.

FIG. 2. Case 1. Second radiograph, taken erect during retching. The duodenal bulb has emptied and its contents appear to have passed back into the stomach; the proximal part of the gastric antrum is tightly contracted; and there has been a shift of barium from the lower into the upper part of the stomach.

FIG. 3. Case 1. Third radiograph, taken erect during emesis. The cardia has become elevated and opened widely; barium is being forced out of the stomach and up the oesophagus; and in the lower part of the stomach there is a long, contracted segment.

FIG. 2. 
quantity of barium, most of which may have come from the duodenal bulb. In the proximal part of the antrum there is a constriction; and the greater curve in this region shows a deep notch and is closely approximated to the lesser curve. Above the notch is a part of the stomach containing hardly any barium, although the lesser and greater curves are widely separated, and it is probable that here the anterior and posterior walls have come into contact with one another. At a higher level, the stomach is filled with barium. The antrum has moved upwards and the pylorus is now level with the lower part of the fourth lumbar vertebra. The gallbladder has also risen and its posteromedial side has become deeply indented by the duodenum.

The third radiograph (Fig. 3), taken just after the second and with the patient's position unchanged, shows the cardia wide open and the lower oesophagus distended with barium. The exposure was made when the barium was being forced out of the stomach and up the oesophagus. Owing to the rapidity of movements which were taking place, some of the shadows are blurred, but it is easy to see big differences between this radiograph and the second one. Although the prepyloric region has hardly changed and seems to contain the same amount of barium as before, the constricted segment above this has greatly increased in length, and a segment where the anterior and posterior walls of the stomach are judged to be in apposition is now shown in the upper part of the body of the stomach. This segment is about the same length as the corresponding segment in the second radiograph, although the position is different. In contrast, the bariumfilled segment above has become much smaller; only the fundus and a small part of the body of the stomach remain filled, and it appears that more than half the quantity of barium originally present has already been expelled.

So far we have not mentioned evidence of diaphragmatic movements in the radiographs. Although very little can be seen of the diaphragm in Fig. 1, its position can be gauged from the relationship between the gastric fundus and the spine, and there was no obvious diaphragmatic movement in the interval between the first two radiographs. But in the short time between the second and third exposures there was a distinct upward movement of the diaphragm and structures closely related to it. The fundus of the stomach rose a distance equal to the height of the body of D12. The cardiac incisura moved upwards a similar distance and at the same time became shallower and wider. The gallbladder rose slightly and the indentation made in it by the duodenum became less marked.

CASE 2 A 21-year-old woman, who had recently had epigastric pain, diarrhoea, and melaena, was being given a barium meal examination in the upright position. She became nauseated as she drank the barium, and after she had finished it, began retching. Screening, which had been interrupted while she drank, was resumed for a brief period, and a radiograph was taken (Fig. 4). This showed all the barium in the fundus and body of the stomach; the pyloric antrum was empty, and no barium had entered the duodenum. After a further spell of fluoroscopy, long enough to show active movement in the antrum but too brief to register it accurately, a second radiograph was exposed (Fig. 5). This showed a little barium in the antrum separated from the main mass of barium by an annular constriction involving both curvatures of the stomach and superficially resembling the pylorus. At this point, the examination was discontinued, but the patient did not actually vomit and the retching soon stopped. The examination of the stomach and duodenum was completed after she had fully recovered, and the appearance of these organs at this stage is shown in Figure 6. No organic lesion was detected.

CASE 3 A 60-year-old housewife had been getting dyspepsia and nausea for two or three months, and examination revealed an indefinite epigastric mass, possibly an enlarged liver. As carcinoma of the stomach seemed a likely diagnosis, she was referred to hospital for a barium meal examination.

This was begun with the patient standing, and she drank about $4 \mathrm{oz}$ of undiluted Micropaque. The oesophagus looked normal and no lesion was seen in the stomach, but gastric emptying did not start immediately. Before any barium had entered the duodenum, the patient was lowered on the $x$-ray table into the supine position. She made no complaint of nausea nor was any retching noticed; but just after the movement of the table stopped, she suddenly vomited, and at this moment, a radiograph was taken (Fig. 7).

The examination was discontinued. On its resumption about 15 minutes later, some barium was seen in the stomach but none in the oesophagus or duodenum, and a second radiograph was taken with the patient supine (Fig. 8). She then began vomiting again, and as her condition was poor, the examination was abandoned.

Interpretation of the radiographs There is no radiographic record of events which preceded the opening of the cardia, but some inferences about the behaviour of the stomach can be drawn from the first radiograph (Fig. 7). Our interpretation of this is as follows. The proximal part of the gastric antrum is empty and invisible, and some barium has been trapped between it and the closed pylorus. The body of the stomach above the empty segment appears to contain a large air bubble. At a higher level, barium is seen filling the gastric fundus and passing up the oesophagus which is widely distended. The site of the hiatus is clearly marked by a deep constriction in the barium shadow, and it may be supposed that the cardia is at the same level. But immediately above this constriction there is a local expansion which is demarcated from the oesophagus above by a shallow constriction. We think it likely that this local expansion was caused by a loculus of stomach above the diaphragm and that the shallow constriction represents the temporarily elevated cardia. If this view is correct, there was transient development of a sliding hiatus hernia when this patient was vomiting.

The second radiograph shows a moderate quantity of barium remaining in the stomach and no evidence of a hiatus hernia. A pool of barium is seen in the fundus, which is situated entirely below the diaphragm, and most of the remainder is in the antrum, the body of the organ 


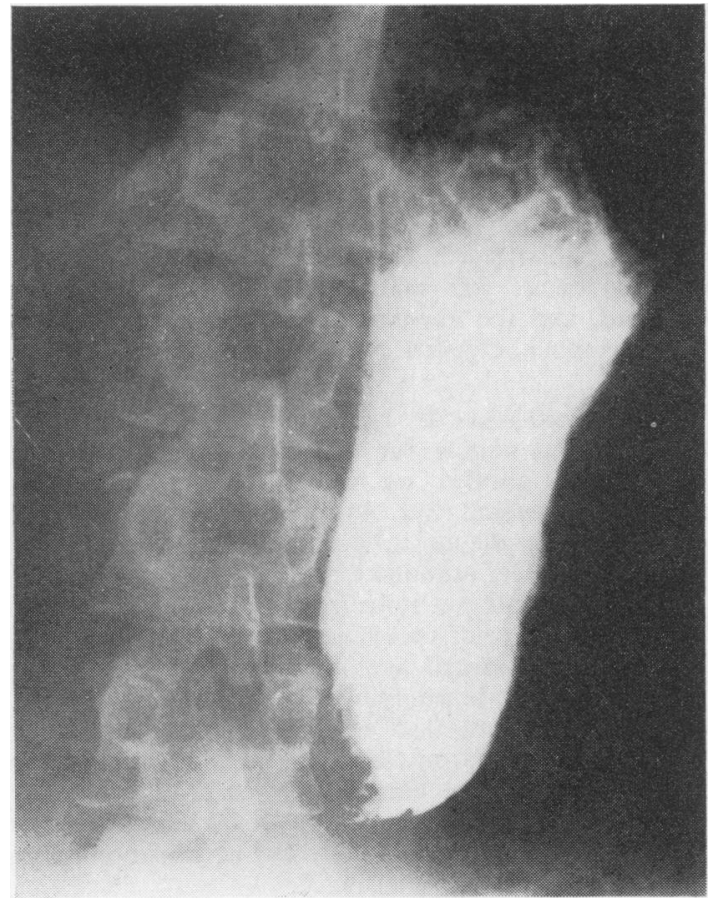

FIG. 4.

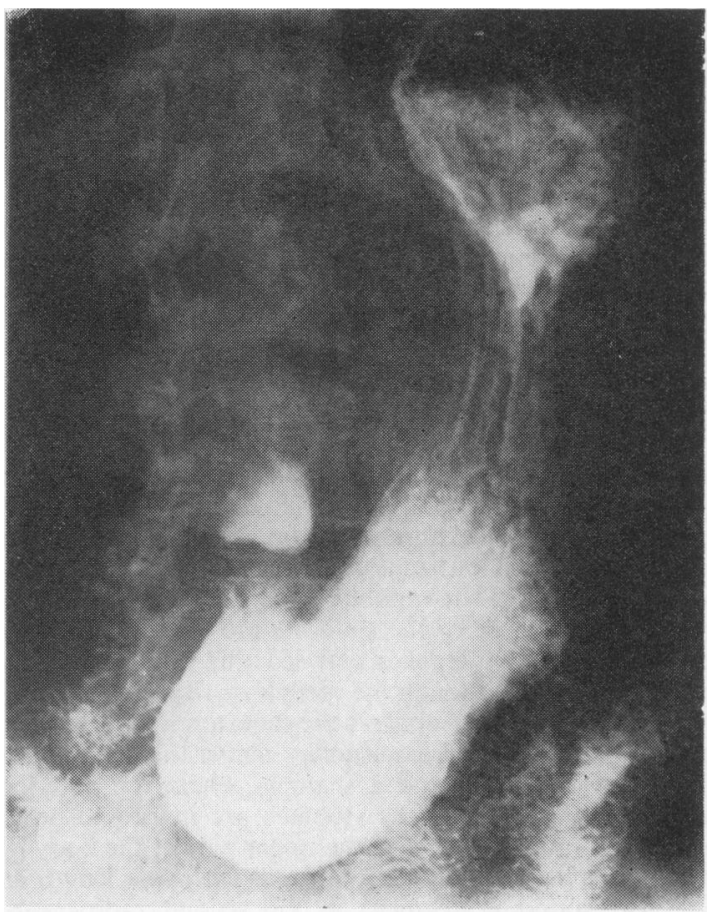

FIG. 6 .

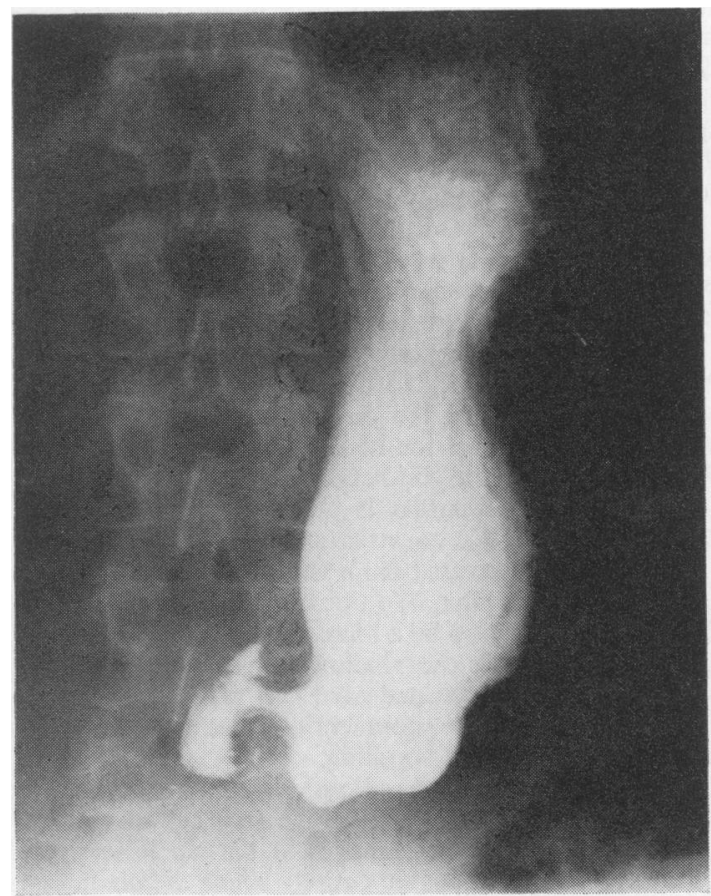

FIG. 4. Case 2. First radiograph, taken erect during retching. The pyloric antrum is contracted and all the barium is in the body and fundus of the stomach.

FIG. 5. Case 2. Second radiograph, taken erect soon after the first, during a period of antral activity. In the antrum there is an annular constriction which resembles the pylorus.

FIG. 6. Case 2. Third radiograph, taken erect after retching movements had ceased and emptying of the stomach into the duodenum had begun.

FIG. 5. 


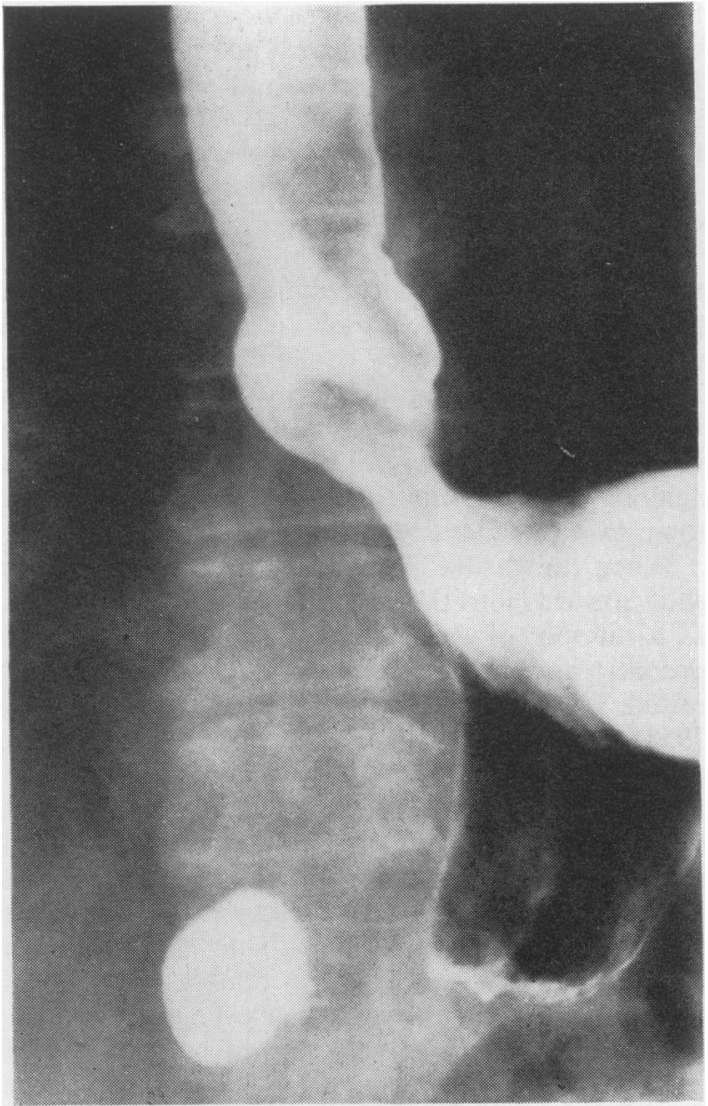

FIG. 7. Case 3. First radiograph, taken supine during emesis. Barium is passing from the gastric fundus up the oesophagus, which is widely dilated, and a sliding hiatus hernia appears to have been temporarily produced.

being almost empty. Only a trace of barium can be seen in a normal-looking, resting oesophagus, and none has entered the duodenum.

\section{DISCUSSION}

We shall discuss our own observations in the light of published studies of vomiting in man as well as experimental work on vomiting in animals such as the cat and dog. In many acts of vomiting three successive stages can be recognized: first nausea, then retching, and finally a stage we shall term 'emesis', in which gastric contents are forcibly expelled from the stomach and brought up to, and out of, the mouth. During these stages, a sequence of movements may be observed in the upper small bowel, stomach, and oesophagus.

NAUSEA Barclay (1936) noted that there was no

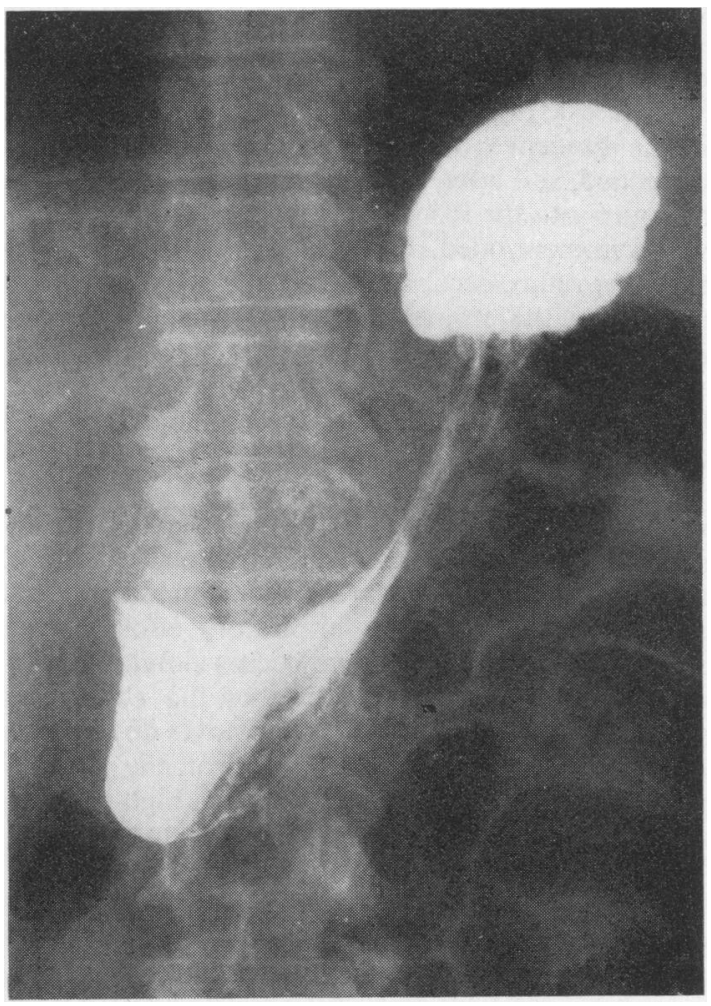

FIG. 8. Case 3. Second radiograph, taken supine after vomiting had ceased. There is a pool of barium in the fundus, which is entirely below the diaphragm, and the body of the stomach is almost empty.

gastric peristalsis during nausea. He also observed a drop in the lowest part of the greater curvature which he attributed to loss of tone in the stomach. We too have seen a sudden descent of this part of the greater curvature in a number of patients when nausea was experienced, but have no radiographic record of such an occurrence.

In contrast to the behaviour of the stomach, the tone of the upper small intestine becomes increased during the stage of nausea. Gregory (1946), reporting on the behaviour of upper loops of jejunum in unanaesthetized dogs after an injection of apomorphine, noted that a stage of nausea was recognizable before retching and vomiting occurred; and during nausea, a brief period when the tone and motility of the bowel were inhibited was followed by an abrupt increase of tone, contractions reappearing to some extent. Nausea in man is frequently accompanied by a generalized contraction of the descending duo- 
denum (Inglefinger and Moss, 1942). Reflux of upper intestinal contents into the stomach before vomiting has also been observed on a number of occasions in man (Barclay, 1936; Alvarez, 1948).

In case 1, at the onset of nausea, the second part of the duodenum was contracted while the bulb was distended, and later, there was reflux of barium from the bulb into the stomach. In the light of the studies we have mentioned, it seems reasonable to assume that this reflux occurred during the stage of nausea and while the stomach was inactive and its tone reduced.

Neither of our other two cases yielded any information about the stage of nausea. This stage ends when retching begins.

RETCHING According to Gold and Hatcher (1926), retching consists of a series of spasmodic and abortive respiratory movements with the glottis closed, during which an inspiratory effort of the chest muscles and diaphragm occurs simultaneously with an expiratory contraction of the abdominal muscles. Although the diaphragm moves downwards violently with each retching movement, the range of movement is small and it remains in a high position throughout this stage of vomiting.

At the same time, movements of the stomach and its contents take place. A detailed description of the movements seen on fluoroscopy in cats has been given by Cannon (1911). First there was total inhibition of the proximal part of the stomach; then there were deep contractions in the distal part; and finally, a strong contraction at the incisura angularis completely divided the stomach into two parts. Somewhat similar observations were made by Hesse (1913) in dogs: first the fundus dilated and remained flaccid, and then the pyloric part of the stomach contracted.

von Czyhlarz and Selka (1913) made fluoroscopic studies of patients vomiting after a bismuth meal and noted that the antrum was fully contracted, while the upper part of the stomach was of normal width. Todd (1930) observed students vomiting after a barium meal; before vomiting, the barium accumulated in the upper part of the stomach, as if it were held up by spasm of the lower part.

These reports together with our own observations have led us to conclude that the stomach is not passive during the act of vomiting but plays an active part.

The view that during retching the pyloric part of the stomach contracts while the upper part is relaxed receives support from gastroscopic observations made by Schindler (1937). In his account of his experiences he has stated: 'Retching usually interferes so much with the examination that it is not possible to observe the behaviour of the stomach, but on two occasions it was possible to do so. Longitudinal folds appeared in the previously smooth antrum and rather quickly thickened, came together, and completely closed the antrum.'

When the cardia opens, retching has ended and emesis begun.

EMESIS It appears that retching culminates in a powerful sustained contraction of the abdominal muscles accompanied by descent of the diaphragm. This raises the intragastric pressure, and in a man vomiting in the upright position, the rise in pressure is sufficient not only to lift the gastric contents against gravity to the mouth but also to accelerate them to a considerable speed.

When the cardia opens, the gastric contents are shot upwards into the gullet. Opening of the cardia, as a number of workers have shown, is an active process; and before it opens, the cardia becomes raised. This elevation has been seen in cats (Torrance, 1958), dogs (Nauta, 1956; Johnson and Laws, 1966), and in man (Inglefinger, 1958).

Johnson and Laws (1966) made a cineradiographic study of the cardia in dogs, with the aid of metal markers, and found that during vomiting the hiatus bulged into the chest and there was herniation of the cardia through the hiatus. They suggested that these movements served the double purpose of tenting the mucosal plug at the cardia and eliminating the abdominal oesophagus, and argued that if the abdominal oesophagus were not eliminated, the rise of pressure within it would nullify the effect of the rise in intragastric pressure. Johnson and Laws also mentioned a chance observation of vomiting in man; pictures were obtained which seemed to show elevation of the cardia into the hiatus with funnelling.

In case 1 , as far as we can judge, the behaviour of the cardia was in accordance with the observations of Johnson and Laws. During emesis there was elevation of the part of the diaphragm in which the hiatus is located, and the cardia not only became elevated but opened so widely that funnelling is an apt description of its appearance. In case 3 , as we have remarked, there may even have been a temporary hiatus hernia. At any rate, we believe that during emesis in both these cases the cardia was elevated and the abdominal oesophagus eliminated.

\section{SUMMARY}

Most of our knowledge of vomiting comes from experimental studies on animals. There have not been many reported studies of vomiting in man.

Chance observations have been made of retching 
and vomiting in patients undergoing routine barium meal examinations, and three cases are reported.

In many acts of vomiting three successive stages can be recognized: nausea, retching, and vomiting. The sequence of movements which occur in the duodenum, stomach, and lower oesophagus, as such an act progresses, has been described.

We are indebted to Dr J. Laws whose advice led to the publication of this paper; to Dr M. J. Turner for permission to include case 3; to $\mathrm{Mr} \mathrm{D}$. R. Floyd and $\mathrm{Dr}$ T. M. Parry for making photographic prints for the illustrations; and to Dr S. C. Truelove who read the typescript and made helpful suggestions.

\section{REFERENCES}

Alvarez, W. C. (1948). An Introduction to Gastro-enterology, 4th ed., p. 484. Hoeber-Harper, New York; Heinemann, London.

Barclay, A. E. (1936). The Digestive Tract, 2nd ed., p. 268. Cambridge University Press, London.
Cannon, W. B. (1911). The Mechanical Factors of Digestion, p. 75. Arnold, London.

von Czyhlarz, E., and Selka, A. (1913). Das röntgenologische Verhalten des Magens bei gastrichen Krisen und beim Brechakte. Wien. klin. Wschr., 26, 842-843.

Gold, H., and Hatcher, R. A. (1926). Studies on vomiting. $J$. Pharmacol. exp. Ther., 28, 209-218.

Gregory, R. A. (1946). Changes in intestinal tone and motility associated with nausea and vomiting. J. Physiol. (Lond.), 105, 58-65.

Hesse, O. (1913). Zur Kenntnis des Brechaktes. Nach Röntgenversuchen an Hunden. Pflüg. Arch. ges. Physiol., 152, 1-22.

Ingelfinger, F. J. (1958). Esophageal motility. Physiol. Rev., 38, 533584 .

- , and Moss, R. E. (1942). The activity of the descending duodenum during nausea. Amer. J. Physiol., 136, 561-566.

Johnson, H. D., and Laws, J. W. (1966). The cardia in swallowing, eructation, and vomiting. Lancet, 2, 1268-1273.

Nauta, J. (1956). The closing mechanism between the oesophagus and the stomach. Gastroenterologia (Basel), 86, 219-232.

Schindler, R. (1937). Gastroscopy, p. 145. University of Chicago Press, Chicago.

Todd, T. W. (1930). Behavior Patterns of the Alimentary Tract. (The Beaumont Foundation Lectures, Series no. 9.) Williams and Wilkins, Baltimore.

Torrance, H. B. (1958). Studies on the mechanism of gastro-oesophageal regurgitation. J. roy. Coll. Surg. Edinb., 4, 54-62. 